\title{
Some Computational Tools for the Analysis of Through Cracks in Stiffened Fuselage Shells
}



the Analysis of Through Cracks in Stiffened Fuselage Shells 


\section{Some Computational Tools for the Analysis of Through Cracks in Stiffened Fuselage Shells}

\author{
C. C. Rankin \& F.A. Brogan \\ Mechanics \& Materials Laboratory \\ Lockheed Missiles \& Space Co. \\ Palo Alto, California, USA
}

\author{
E. Riks \\ Faculty of Aerospace Engineering \\ Technical University of Delft \\ Delft, The Netherlands
}

\section{Summary}

Reliable analytical methods that predict the structural integrity and residual strength of aircraft fuselage structures containing cracks are needed to help to understand the behavior of pressurized stiffened shells with damage, so that it becomes possible to determine the safe life of such a shell. Of special importance is the ability to determine under what conditions local failure, once initiated, will propagate. In this paper we shall present a reliable and efficient method for computing the energy release rate for cracks of varying length in a typical stiffened metallic fuselage under general loading conditions. The models used in the simulation are derived from an extensive analysis of a fuselage barrel section subjected to operational flight loads. Energy release rates are computed as a function of the length of the crack, its location, and the crack propagation mode.

\section{Introduction}

The behavior of through cracks in pressurized fuselages, Figure 1, is believed to be dominated by three influence factors - if we ignore a fourth: plasticity effects at the crack tips. The first is of a geometrically nonlinear origin, e.g., Lemaitre, Turbat and Loubet (1977), Riks and den Reijer (1987), Riks (1978) and Ansell (1988), Atluri and Tong (1989), Chen (1991), Chen and Schijve (1991). It is a stiffening effect that is caused by tensile membrane stresses in the direction parallel to the crack faces. By restricting the so called bulging and opening of the crack, these stresses reduce the intensity of the stress singularity at the crack tip significantly.

A second factor of importance is the geometry and material properties of the surrounding superstructure i.e., the influence of the presence of the stiffeners and bulkheads Riks (1987) and Ansell (1988). These fixtures cause significant local changes in the barrel stresses of the shell under pressure, thus influencing the loading of the crack. 


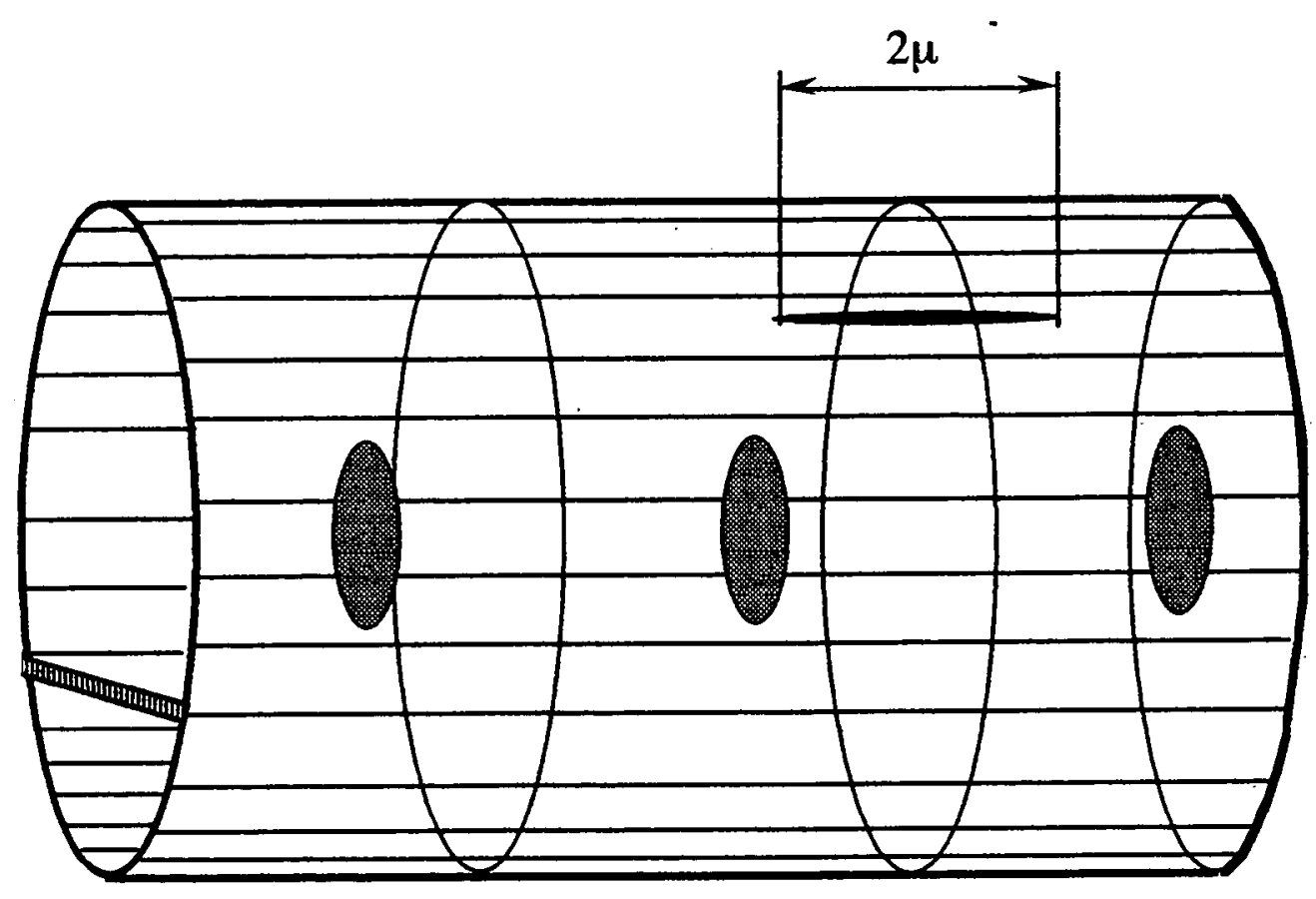

Figure 1

Finally, we should mention the influence of the secondary loads (in addition to the pressure load) on the fuselage that also give rise to changes.

As we have shown on previous occasions Riks, Rankin and Brogan (1989) and (1992), the finite element method has a great potential for dealing with a problem of this complexity. In this report, we shall present a further development of special tools for the analysis cracks in fuselage shells that we have implemented in the STAGS program: Almroth, Brogan and Stanley (1983). In particular we shall describe a method for the calculation of the energy release rates of cracks that may be useful to determine the damage tolerance of a structure containing a crack.

An interesting and important question in the assessment of the damage tolerance of metallic fuselage shells is the capability to predict the way the crack is growing at each particular instant of the crack growing process. Will it grow in a self similar fashion or will it kink or bifurcate? Alternatively, will the crack turn away from its original growing direction in a gradual fashion (in the present context of pressurized cylindrical shells, this is also called crack flapping)?

Theories about the growth of a crack in a plate under pure membrane stress conditions (i.e., no bending) have been proposed by several authors of whom we mention Finnie and Saith (1973), Leevers, Radon and Culver (1977), Cotterell and Rice (1980), Sumi, Nemat Nasser and Keer (1985), Ramulu, Kobayashi and Kang (1982), Ramulu and Kobayashi (1983). 
These theory's state criteria under which a growing crack tends to deviate from its previous growing direction. Although there is not much unanimity on the precise terms of these criteria, the main stream of thought seems to converge to the following observations, Zaal (1992).

(i) Crack propagation occurs in such a way that the condition $\mathrm{K}_{\mathrm{II}}=0$ at the crack tip is maintained. (Here $\mathrm{K}_{\mathrm{II}}$ denotes the stress intensity factor related to the shearing mode of cracking.)

(ii) If $\mathrm{K}_{\mathrm{II}} \neq 0$, the crack tends to kink under finite angle for $\mathrm{K}_{\mathrm{II}}>\mathrm{k}^{*}$ where $\mathrm{k}^{*}$ is a certain threshold value that is material dependent.

(iii) If $K_{I I}=0$, the growth of the crack will be self similar but only when an additional criterion is fulfilled. If this condition is violated the crack will turn away from the original direction with finite curvature. About the precise terms of this special condition no agreement has yet been reached. There is, however, considerable theoretical evidence that it should concern the so called $T$ - stress which is a basic non-singular component of the stress field that surrounds the crack tip. ${ }^{\text {) }}$

A complicating factor in this set of rules is that in a pressurized shell the state of stress around the crack tip can never be purely membrane. There will always be bending components present and it is at this moment not quite clear in what way the above criteria have to be changed to account for the combined action of membrane and bending stresses. In summary, the theory is still incomplete and the correlation with experimental evidence is not entirely satisfactory.

It follows from these observations that a modest but still significant step towards crack path prediction consists of a means to verify whether criterion (i) is fulfilled or not. Such verification, as it turns out, can be done with a very simple and practical method that makes use of a node release procedure. (The energy release rates are in some sense equivalent to the stress intensity factors.) The method that we will describe in this paper computes the energy release rates of a crack in terms of separate components that correspond to the various modes of cracking the crack tip is experiencing. It can be applied even when the crack is located in a parts of the shell with irregular attributes such as stiffeners. We believe that the method should be useful in the assessment of the damage tolerance of stiffened fuselage shell structures. It may also be useful in the assessment of the crack growing process under fatigue loading conditions.

1) We notice here that the first rule (i) given above can be seen as a necessary condition for self similar crackgrowth. Condition (i) and (iii) combined would then represent the necessary and sufficient condition for self similar crack growth. 


\section{Equilibrium models for cracked shells}

\subsection{Basic assumptions}

The fuselage shell will be modeled in such a way that the resulting equations approximate the equations of classical nonlinear shell theory (see for instance Koiter (1966)). This theory is based on two fundamental assumptions: (i) The state of stress in the shell is governed by linear elasticity. (ii) The state of stress is approximately plane.

This means that the crack problem will be treated as a plain stress problem which is a gross simplification of the actual state of stress at the crack tip. Please note that the nonlinearity referred to in this paper is purely of a geometrical nature.

It is well known that the finite element discretization process of the shell model produces a set of nonlinear equations:

$$
f(d ; \lambda ; m)=0
$$

The nonlinear vector function $f \in R_{N}$ is usually of a large dimension. The argument $d \in R_{n}$ stands for the deformation variables that describe the state of deformation of the structure. More specifically, $d$ is the collection of the nodal variables of the finite element method used. The parameter $\lambda$ is the loading intensity, and the parameter $\mu$ represents the length of the crack.

(Notice that we will follow the convention of defining the actual crack length by $2 \mu$ which is the actual cord length of the fracture path, see figure 1).

\subsection{Solution strategy}

To analyze a crack in a fuselage under pressure it is first necessary to obtain the state of deformation of the structure under its service load $\lambda=\lambda_{1}$. This state of deformation: $d=d\left(\lambda_{1}, \mu\right)$ is determined by the set of equations (2.1), the discrete analog of the shell equations. To compute it we use path-following methods (also called continuation methods). We will not go into details here because the basic theory of these methods is now well established; interested readers should consult for example: Riks (1984), Riks (1987) and, or the references therein.

As with most finite element codes for nonlinear analysis, the STAGS program that we use here is equipped with a basic path following procedure (see for a description, Riks (1987)). It is used to compute the loading paths defined by:

$$
\begin{aligned}
& 0 \leq \lambda \leq \lambda_{\text {service }} ; \mu=\mu_{1} \\
& 0 \leq \lambda \leq \lambda_{\text {service }} ; \mu=\mu_{2}
\end{aligned}
$$

which are obtained in terms of a series of solution points $d_{i}$ that belong to a series of load steps $\lambda_{i}: i=1,2,3, \ldots, \ldots . .$. . Two of those paths (I and III) are, for instance, given in figure 2 . The figure illustrates, in a simplified way, the solutions we seek in the space of the deformation 
variables versus the load $\lambda$ and crack length $\mu$. It should be noted, however, that the objective of the analysis is seldom restricted to one particular crack length or one particular crack path. It frequently occurs that many crack geometries must be investigated. The most common situation is depicted in the figure by the path II that is defined by:

$$
\mu_{1} \leq \mu \leq \mu_{2} ; \lambda=\lambda_{\text {service }}
$$

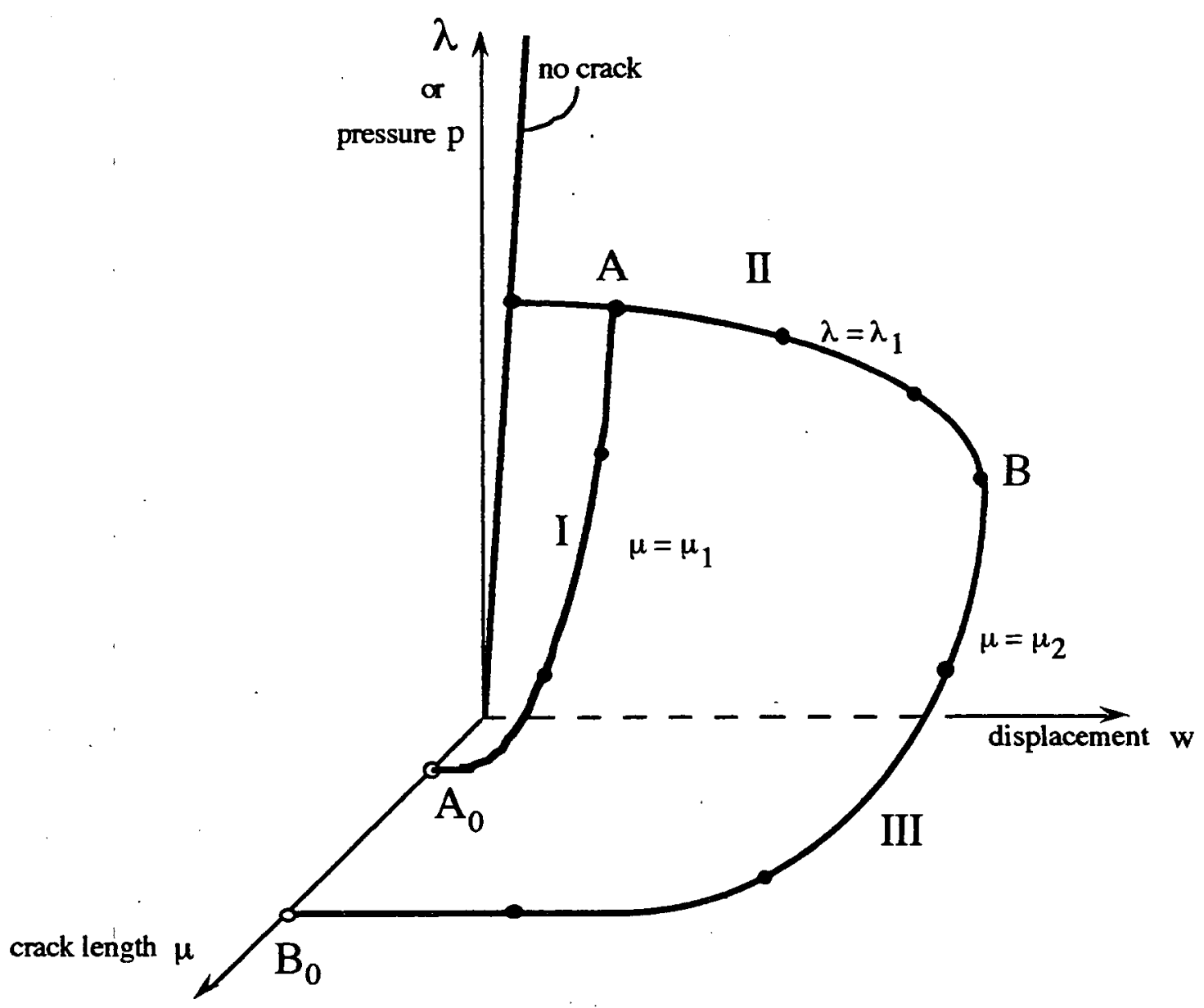

Figure 2

In other words, one needs a method for computing the successive equilibrium states of cracks with increasing crack length, or the other way around. The procedure that accomplishes this task in STAGS is called "load relaxation procedure". It is a specialized form of a path following technique that will be discussed in some detail in section 3 .

2.4 Energy release rates and modes of cracking

The global form of the energy release rate is defined as: 


$$
\mathrm{G}=-\frac{\mathrm{dP}}{\mathrm{hd} \mu}=-\operatorname{LIM}_{\Delta \mu \rightarrow 0}\left\{\frac{\Delta P}{\mathrm{~h} \Delta \mu}\right\}
$$

where $P$ is the total potential energy of the structure as mentioned above and d $\mu$ refers to a crack advance that is tangential to the crack path at the tip and $h=$ the thickness of the shell at the crack tip.

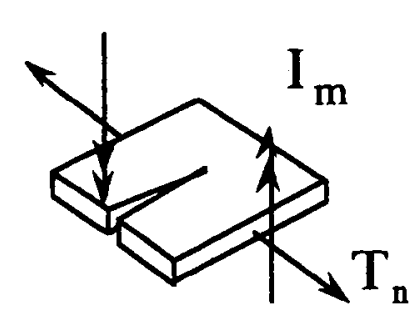

\section{Membrane}
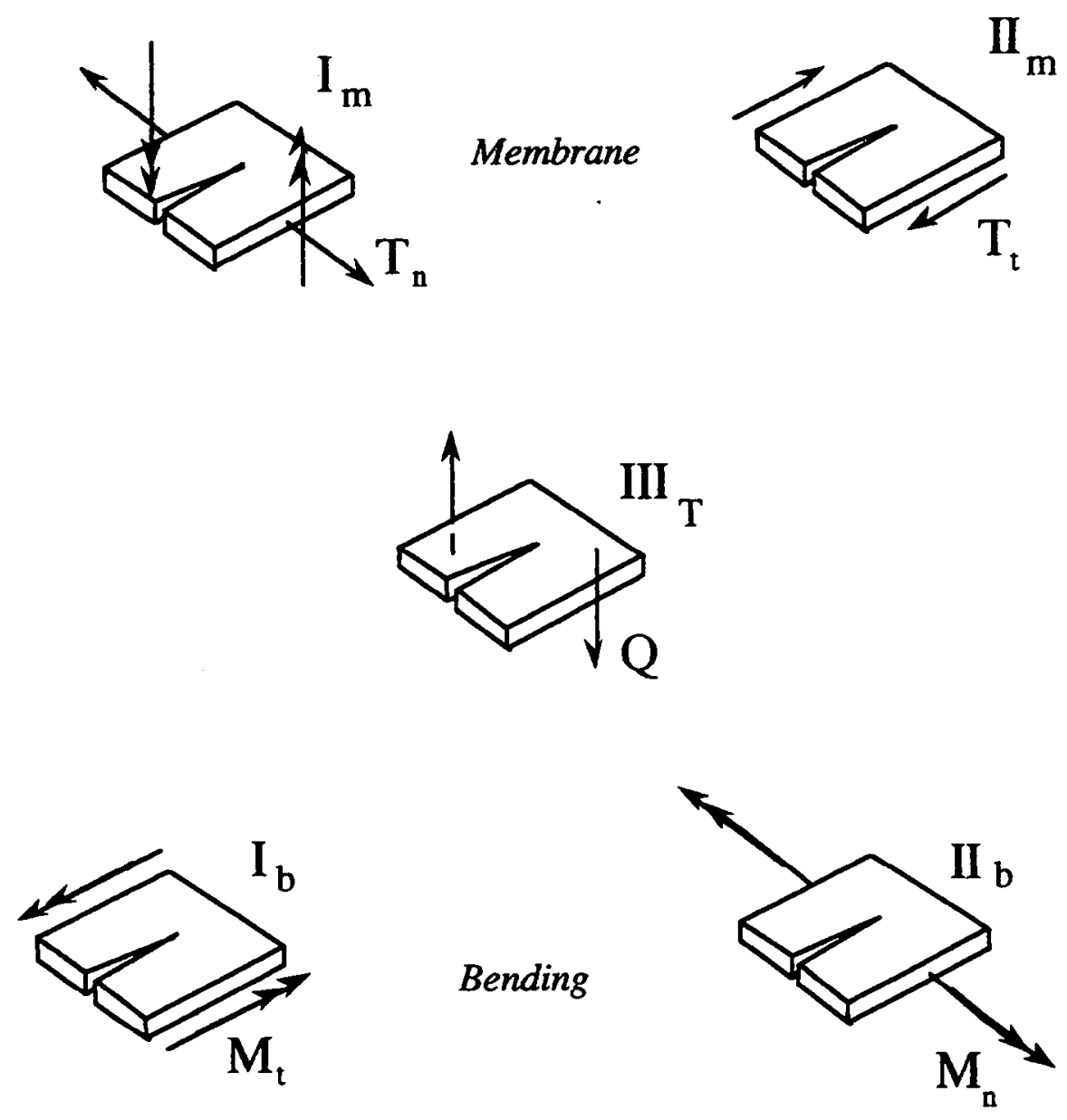

Figure 3 Modes of Cracking

In chapter 3 we will compute the energy release directly by means of the release of a node in front of the current crack. This procedure makes use of the energy conservation principle which states that the change of the total potential energy of the structure invoked by the node release process is equal to the work expended during this process.

As a mechanism to let a crack grow, node release offers a natural way to decompose the energy release rate into separate components that represent the various modes of deformation the crack tip is subjected to. For the quadrilateral shell finite elements that we use here, there are six degrees of freedom associated with the motion of the node, three translations and three rotations. This is unusual because most shell finite elements do not include the third component, 
i.e., the drilling degree of freedom in the formulation. The description of the shell element is given in: Rankin and Brogan (1991).

We mark the two nodes that involved in the separation process by superscripts (1) and (2). If the rates of change of the displacements (in terms of the nodal degrees of freedom) that describe the subsequent motion of these nodes during the release process are denoted by $v_{i}^{1}, v_{i}$ $(i=1,2,3)$ and if, similarly, the angular velocities are denoted by $v_{i}^{1}, v_{i}(i=4,5,6)$, the energy release during the separation process of the node is given by:

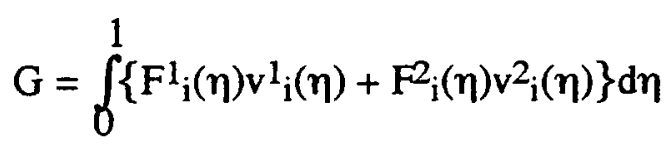

The parameter $\eta$ is here the path parameter that describes the evolution of the opening process.

It should be mentioned at this point that the effective resultants $F^{1}$ and $F^{2}$ that make up the dynamic boundary conditions in classical shell theory involve only four independent quantities. These are two effective membrane forces and two effective bending moments, or, alternatively, two effective membrane forces combined with a bending moment and an effective transverse force in the direction of the shell normal. However, in this paper we have not followed this convention, because, among others, the stress field at the crack tip cannot be represented in the context of these assumptions of shell theory. Instead, we introduce the five contributions associated with the mechanical modes of cracking portrayed in figure 3 . They are further discussed in chapter 3 where we describe the details of their computation.

\section{Crack Analysis Tools}

3.1 Node release and node closure as a continuation process

We will now discuss a node release and a node closure procedure that are very useful for the analysis of through cracks in shells. Conceptually, these techniques are remarkably simple considering how powerful they are in practical applications. To describe them, we should first mention that the cracks that we model here are always aligned along a string of contiguous nodes. The fracture path in front of the current crack is then also a contiguous string of nodes which are considered to be doublets, i.e., they are viewed as double nodes that occupy the same position in space. Of course, we use here the well-known concept of master and slave nodes.

The opening procedure involves a sequence of nodes along the fracture path in front of the current crack. The closure procedure involves two strings of nodes on either side of the crack faces behind the current crack front. These processes are nonlinear, in principle, so that they must be carried out with a continuation procedure. Please note that the two opposing motions as illustrated in figure 4 correspond to the case that the opening and closing process involves a single node step only. 


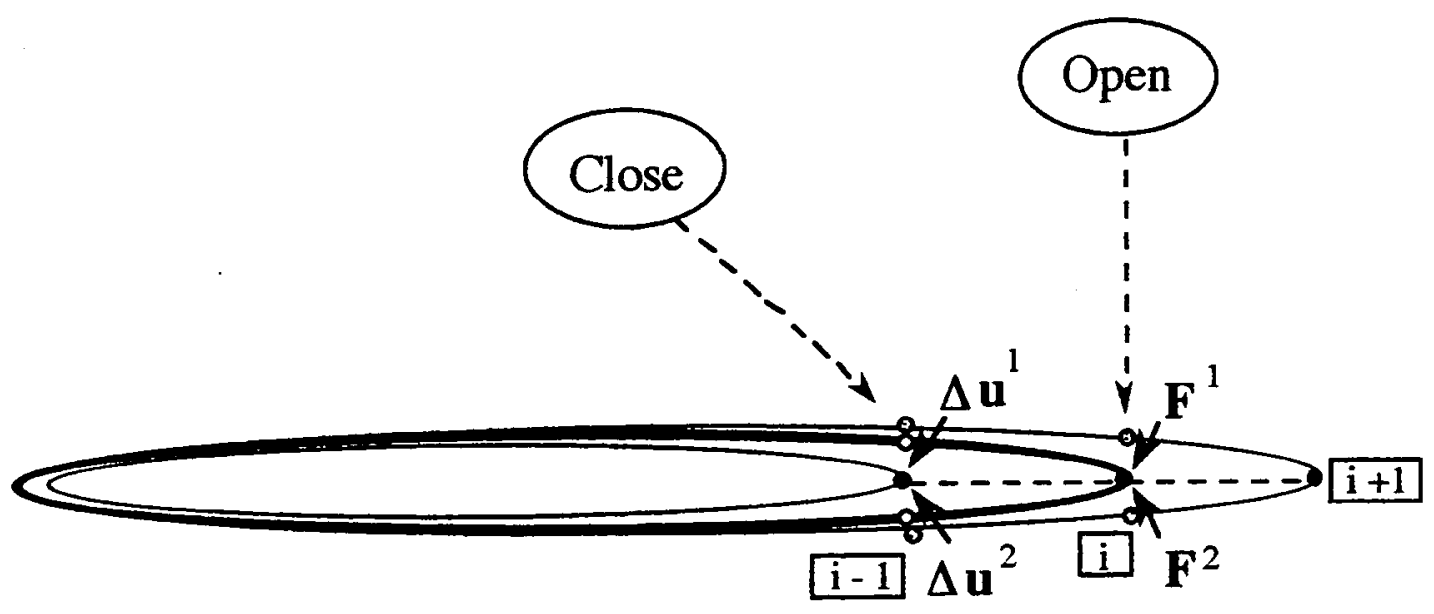

Figure 4 The opening and closing process

\subsection{Formulation of the opening process}

The opening process is straightforward. The user selects a string of nodes along the projected crack path. The deformed shape belonging to the shorter crack is denoted by $d_{0}=d\left(\lambda, \mu_{0}\right)$ where, as before, $\lambda$ denotes the load intensity at which $d_{0}$ is obtained and $\mu_{0}$ characterizes the current length of the crack. The string of nodes that are selected will increase the length of the crack form $\mu_{0}$ to $\mu_{e}$ and the solution that belongs to the new length $\mu_{e}$ will be denoted by $d(\lambda$, $\left.\mu_{e}\right)$. Because we are releasing nodes, the dimension of the displacement vector $d_{0}$ is not equal to the dimension of $d_{\mathrm{e}}$. The increase of the number of degrees of freedom equals the number of nodes involved in the opening process times the number of degrees of freedom of each node.

Let the set of freedoms that corresponds to the master nodes be denoted by $\mathbf{u}_{1}$ and the one that corresponds to the slaves by $\mathbf{m}_{2}$. Then we can write for the two solutions $d_{0}$ and $d_{e}$ :

$$
\begin{aligned}
& \mathbf{d}_{0}=\left(\begin{array}{l}
\mathbf{t} \\
\mathbf{u}_{1}
\end{array}\right) \\
& \mathbf{d}_{\mathbf{e}}=\left(\begin{array}{l}
\mathbf{t} \\
\mathbf{u}_{1} \\
\mathbf{u}_{2}
\end{array}\right)
\end{aligned}
$$

where $t$ denotes the set of degrees of freedom of the nodes not involved in the opening process. Together with these vectors of degrees of freedom $t, \mathbf{u}_{1}$ and $\mathbf{u}_{2}$, there are the vectors $\mathbf{r}, \mathbf{r}_{1}$ and $\mathbf{r}_{2}$ of nodal forces. Associated with these quantities is the virtual work expression: 


$$
\delta A=\mathbf{r} . \delta \mathbf{t}+\mathbf{r}_{1} . \delta \mathbf{u}_{1}+\mathbf{r}_{2} . \delta \mathbf{u}_{2}
$$

where $\mathbf{a} \cdot \mathbf{b}$ is the notation for the inner product of two vectors $\mathbf{a}$ and $\mathbf{b}$.

Now it is clear that for a crack with length $\mu_{0}$ :

$$
\mathbf{r}=0 ; \mathbf{r}_{1}+\mathbf{r}_{2}=0 ; \delta \mathbf{u}_{1}=\delta \mathbf{n}_{2}
$$

while for the crack with the new length $\mu_{\mathrm{e}}$ we must have:

$$
\mathbf{r}=\mathbf{r}_{1}=\mathbf{r}_{2}=0 \quad ; \delta \mathbf{u}_{1} \neq \delta \mathbf{u}_{2}
$$

The nodal forces acting on the master and slave nodes in state are thus in equilibrium in the configuration (1), while they are reduced to zero for ${ }^{(2)}$.

\section{Equilibrium equations for the shorter crack}

The equilibrium state of the cracked structure with crack length $\mu_{0}$ is determined by the set of equations:

$$
\frac{\partial \mathrm{P}}{\partial \mathbf{t}}=0 ; \frac{\partial \mathrm{P}}{\partial \mathbf{u}_{1}}=0
$$

where $P$ is the potential energy of the cracked structure in the configuration (1):

$$
\mathrm{P}=\mathrm{P}\left(\mathbf{t}, \mathbf{u}_{1} ; \lambda\right)
$$

This is a formulation of the equations for the shorter crack $\mu_{0}$, where the slave degrees of freedom are thought to be eliminated. An equivalent formulation of the same problem can be obtained via a formal change in the model of this crack. We now declare the slave nodes free and define the modified potential energy for this case:

$$
\mathrm{P}^{\#}=\mathrm{P}^{\sim}\left(\mathbf{t}, \mathbf{u}_{1}, \mathbf{u}_{2}, \lambda, \mu_{0}\right)-\mathbf{r}_{1} \cdot \mathbf{u}_{1}-\mathbf{r}_{2} \cdot \mathbf{u}_{2}
$$

The nodal forces $\mathbf{r}_{1}$ and $\mathbf{r}_{2}$ defined at (3.5) are now used as an additional loading system that keeps the two node systems (1) and (2) closed. The equilibrium equations for this modified model read:

$$
\frac{\partial \mathrm{P}^{\#}}{\partial \mathbf{t}}=\frac{\partial \mathrm{P}^{\sim}}{\partial \mathbf{t}}=\mathbf{0}
$$




$$
\frac{\partial \mathrm{P}^{\sim}\left(\mathbf{d}, \lambda, \mu_{0}\right)}{\partial \mathbf{u}_{1}}-\mathbf{r}_{1}=0 ; \frac{\partial \mathrm{P}^{\sim}\left(\mathbf{d}, \lambda, \mu_{0}\right)}{\partial \mathbf{u}_{2}}-\mathbf{r}_{2}=0
$$

where the nodal forces are defined by the derivatives:

$$
\mathbf{r}_{1}=\frac{\partial \mathrm{P}^{\sim}\left(\mathbf{d}_{0}, \lambda, \mu_{0}\right)}{\partial \mathbf{u}_{1}} \quad \mathbf{r}_{2}=\frac{\partial \mathrm{P}^{\sim}\left(\mathbf{d}_{0}, \lambda, \mu_{0}\right)}{\partial \mathbf{u}_{2}}
$$

which are evaluated at the equilibrium solution $\mathbf{d} 0$.

It should be clear that the latter formulation interprets model with crack length $\mu=\mu_{0}$ as a problem whereby a structure with a crack of length $\mu=\mu_{\mathrm{e}}$ is closed over a trajectory of length: $\Delta \mu=\mu_{e}-\mu_{0}$ by means of two sets of equal but opposing forces $\mathbf{r}_{1}$ and $\mathbf{r}_{2}$.

\section{Equilibrium equations for the longer crack}

Consider now the equilibrium equations that determine the deformation of the structure with the longer crack $\mu_{\mathrm{e}}$. They are given by:

$$
\frac{\partial \mathrm{P}^{\sim}}{\partial \mathbf{t}}=0 ; \frac{\partial \mathrm{P}^{\sim}}{\partial \mathbf{u}_{1}}=0 ; \frac{\partial \mathrm{P}^{\sim}}{\partial \mathbf{u}_{2}}=0
$$

where the potential energy function $\mathrm{P}^{\sim}$ is identical to the function $\mathrm{P}^{\sim}$ in (3.8).

\section{The connection between the two sets of equations}

Comparing equations (3.9) with (3.10) we observe that they both belong to the family of problems defined by:

$$
\frac{\partial \mathrm{P}^{\sim}}{\partial \mathbf{t}}=0 ; \frac{\partial \mathrm{P}^{\sim}}{\partial \mathbf{u}_{1}}-(1-\zeta) \mathbf{r}_{1}=0 ; \frac{\partial \mathrm{P}^{\sim}}{\partial \mathbf{u}_{2}}-(1-\zeta) \mathbf{r}_{2}=0
$$

for parameter values of $\xi$ in the interval: $0 \leq \zeta \leq 1$. The last set of equations defines a path of solutions that connect the states (1) and (2) in a continuous manner. It can be seen as a loading process whereby a subset of the loading on the structure is released from intensity $(1-\zeta)=1$ in state (1) to intensity $(1-\zeta)=0$ in state (2); hence the name "load relaxation method."

The node relaxation method is thus equivalent to the computation of a deformation path of a structure under a varying load. It can therefore readily be cast into the framework of the standard path following techniques that are available in most nonlinear finite element systems. This is also the way it is done in the STAGS code. 


\subsection{Closure of the crack}

It may occur that the analysis requires the closure of a crack rather than the opening, for purely computational reasons, for example. Such a process is the reverse of the process described in the previous section, and it requires a formulation that can be seen as the mirror image of the that given for the release process. We will also describe this formulation here, although it has not yet been implemented in the STAGS program.

A slight difficulty here is that the position the two nodes take after they have been unified is not known in advance. It is expedient therefore to replace the energy function given in (3.8), for the crack with length $\mu^{*}$, by:

$$
\mathrm{P}^{\#}=\mathrm{P}^{\sim}\left(\mathbf{t}, \mathbf{u}_{1}, \mathbf{u}_{2}, \lambda, \mu^{*}\right)+\mathbf{r} \cdot\left[\mathbf{u}_{1}-\mathbf{u}_{2}-(1-\xi) \Delta \mathbf{u}^{0}\right]
$$

where $\mathbf{r}$ is a set of Lagrange multipliers that is connected with the constraint condition:

$$
\mathbf{u}_{1}-\mathbf{u}_{2}-(1-\xi) \Delta \mathbf{u}^{0}=0
$$

which is identified as the set of forces that is necessary to bring to the nodes together that are involved in the closing operation. The value $\Delta \mathbf{u}^{0}=\mathbf{u}_{1}(\zeta=0)-\mathbf{u}_{2}(\xi=0)$ corresponds here to the difference of the two sets of displacements $\mathbf{u}_{1}$ and $\mathbf{u}_{2}$ before the two sets of nodes are brought together. The formulation (3.11) is thus equivalent to (3.6), (3.7) for $\zeta=0$. When the "loading parameter" $\zeta$ is varied from 0 to 1 , (3.12) represents the definition of a path of solutions from the opened to the closed situation.

Note that the variation of (3.12) with respect to $\mathbf{t}, \mathbf{u}_{1}, \mathbf{u}_{2}$ and $\mathbf{r}$ will lead to the set of equations

$$
\frac{\partial \mathrm{P}^{\#}}{\partial \mathbf{t}}=0 ; \frac{\partial \mathrm{P}^{\#}}{\partial \mathbf{u}_{1}}=0 ; \frac{\partial \mathrm{P}^{\#}}{\partial \mathbf{u}_{2}}=0 ; \frac{\partial \mathrm{P}^{\#}}{\partial \mathbf{r}}=0
$$

or

$$
\frac{\partial \mathrm{P}^{\sim}}{\partial \mathbf{t}}=0 ; \frac{\partial \mathrm{P}^{\sim}}{\partial \mathbf{u}_{1}}+\mathbf{r}=0 ; \frac{\partial \mathrm{P}^{\sim}}{\partial \mathbf{u}_{2}}-\mathbf{r}=0 ;\left(\mathbf{u}_{1}-\mathbf{u}_{2}-\xi \Delta \mathbf{u}^{0}\right)=0
$$

If the dimension of the set $\mathbf{t}$ is $\mathbf{N}$, and that of the nodal displacements $\mathbf{u}_{1} \& \mathbf{u}_{2}$ is

$(k+k)$, then the dimension of the set of computational degrees of freedom is $(N+3 k)$ :

$$
\mathbf{x}=\left(\begin{array}{c}
\mathbf{t} \\
\mathbf{u}_{1} \\
\mathbf{u}_{2} \\
\mathbf{r}
\end{array}\right) \in \mathbf{R}_{N}+3 k
$$

It is again possible to formulate a continuation process that computes the solution $\mathbf{x}$ as a function of $\zeta$ on the basis of the set of equations (3.13) just as this was possible in the case of 
the opening process that we discussed earlier. It turns out, however, that a direct implementation of this strategy is not the most convenient way of approaching the solution of the closure problem. It is better to remove first the reaction force system $\mathbf{r}$ (the connection forces) and the slave displacement $\mathbf{u}_{2}$ from the equation set. This operation results in two sets of equations:

$$
\frac{\partial \mathrm{P}^{\sim}}{\partial \mathbf{t}}=0 ; \quad \frac{\partial \mathrm{P}^{\sim}}{\partial \mathbf{u}_{1}}+\frac{\partial \mathrm{P}^{\sim}}{\partial \mathbf{u}_{2}}=0
$$

and where $\mathbf{u}_{2}$, which occurs in these equations, is considered to be prescribed by:

$$
\mathbf{u}_{2}=\mathbf{u}_{1}-\zeta \Delta \mathbf{u}^{0}
$$

Equations $(3.14+3.15)$ correspond to a set of equations of the form:

$$
\begin{gathered}
\mathbf{f}\left(\mathbf{t}, \mathbf{u}_{1} ; \zeta\right)=0 \quad \mathbf{g}\left(\mathbf{t}, \mathbf{u}_{1} ; \zeta\right)=0 \\
\mathbf{f}=\frac{\partial \mathrm{P}^{\sim}}{\partial \mathbf{t}} ; \mathbf{g}=\frac{\partial \mathrm{P}^{\sim}}{\partial \mathbf{u}_{1}}+\frac{\partial \mathrm{P}^{\sim}}{\partial \mathbf{u}_{2}}
\end{gathered}
$$

where $\xi$ is the loading parameter in terms of a prescribed displacement. The continuation process that we had in mind can now be formulated for the equations in the reduced form. This will produce the path :

$$
\mathbf{d}=\left(\begin{array}{c}
\mathbf{t}(\zeta) \\
\mathbf{u}_{1}(\zeta)
\end{array}\right) \quad \text { for } \zeta=0 \rightarrow 1
$$

The end point of this set is the desired solution for the shorter crack.

\subsection{Computation of the energy release rates}

The discrete analog of the $\mathrm{J}$ - integral method e.g.,: Parks (1974), Hellen (1975), deLorenzi (1985) and Hellen (1987) is a useful and accurate tool for the computation of energy release rates of through cracks in shells as was demonstrated in: Riks, Rankin and Brogan (1989), (1991). However, if one wants to differentiate between the contributions of the different modes of cracking, this method needs considerable adaptation. Moreover, it is difficult to see how the method would be applied when there are irregular structural components in the neighborhood of the crack tip such as a stiffener or a doubler. An alternative method, which is simple and which is capable of distinguishing between the contributions of each mode of cracking, is the direct computation of the energy release by node release. The method makes use of the opening process discussed earlier.

The energy release associated with the advance of the crack from node (i) to node $(i+1)$ (the forward movement) is given by the integral: 


$$
G_{f}=-\int_{0}^{1} \mathbf{r}^{1}(\zeta) d\left[\mathbf{u}^{1}(\xi)\right]-\int_{0}^{1} \mathbf{r}^{2}(\zeta) d\left[\mathbf{u}^{2}(\zeta)\right]
$$

whereby the integration is carried out along the continuation path (parametrized by $\zeta$ ) and the values $\zeta=0$ and $\zeta=1$ indicate the begin and end point of the path. Consider here $\mathbf{a}^{1}$ as the displacement of the master node of (i) ${ }^{1}$ and $\mathbf{r}^{1}$ the force on this node. The corresponding displacement and force on the slave node (i) ${ }^{2}$ are denoted by $\mathbf{u}^{2}$ and $\mathbf{r}^{2}$.

The closure of the crack by forcing the two nodes $(i-1)^{1}$ and $(i-1)^{2}$ to occupy the same position in the three dimensional space $\mathbb{E}_{3}$ requires work that is added to the structure. This work is the reverse of the energy release if we had opened the crack from nodes $(i-1)$ to (i). Thus, in the case of closure (the backward movement) we compute the energy release from:

$$
\mathrm{G}_{\mathrm{b}}=\int_{0}^{1} \mathbf{r}^{1}(\zeta) \mathrm{d}\left[\mathbf{u}^{1}(\zeta)\right]+\int_{0}^{1} \mathbf{r}^{2}(\xi) \mathrm{d}\left[\mathbf{u}^{2}(\zeta)\right]
$$

The value $\zeta=0$ and $\zeta=1$ correspond here to the opened and closed situation respectively. It is the reverse of the previous calculation at (3.18).

As mentioned before, in the present implementation version of the method in STAGS, the energy release is computed in a forward step only. The release rate in that case corresponds to a crack of a length that is in between the two nodes that are involved in the opening process. Thus the release rates are computed for a crack of the length $\mu=\frac{1}{2}\left[\mu_{0}+\mu_{e}\right]$.

When the distance between the nodes that are released is small, the calculation is particular simple. Let the increase of the displacements and rotations of the master and slave nodes be denoted by $\Delta u_{i}$ and $\Delta u_{i}^{2}$ respectively $(i=1,2, . ., 6)$. The five contributions are then computed from the linearization of (3.18):

$$
\begin{aligned}
& I_{m}: \quad G_{1}=\frac{1}{2 h b}\left[r_{2} \Delta u^{1}{ }_{2}-r_{2} \Delta u^{2}{ }_{2}+r_{6} \Delta u^{1}{ }_{6}-r_{6} \Delta u^{2}{ }_{6}\right] \\
& \mathrm{II}_{\mathrm{m}}: \quad \mathrm{G}_{2}=\frac{1}{2 \mathrm{hb}}\left[\mathrm{r}_{1} \Delta \mathrm{u}_{1} \mathrm{I}_{1}-\mathrm{r}_{1} \Delta \mathrm{u}^{2}{ }_{1}\right] \\
& \text { III: } \quad \mathrm{G}_{3}=\frac{1}{2 \mathrm{hb}}\left[\mathrm{r}_{3} \Delta \mathrm{u}_{3}-\mathrm{r}_{3} \Delta \mathrm{u}^{2}{ }_{3}\right] \\
& \mathrm{I}_{\mathrm{b}}: \quad \mathrm{G}_{4}=\frac{1}{2 \mathrm{hb}}\left[\mathrm{r}_{4} \Delta \mathrm{u}_{4} 1_{4}-\mathrm{r}_{4} \Delta \mathrm{u}^{2}{ }_{4}\right] \\
& \mathrm{II}_{\mathrm{b}}: \quad \mathrm{G}_{5}=\frac{1}{2 \mathrm{hb}}\left[\mathrm{r}_{5} \Delta \mathrm{u}^{1}{ }_{5}-\mathrm{r}_{5} \Delta \mathrm{u}^{2} 5\right]
\end{aligned}
$$


Note that this representation neglects the fact that the nodal forces $r_{1}$ are nonlinearly dependent on the distance between the two nodes that are involved in the separation process.

\subsection{A note on the accuracy of the node release method.}

The node release method corresponds to numerical differentiation of the energy with respect to an advance of the crack front in a direction tangent to the crack path at the crack length $\mu$. Instead of the differential, we use the finite difference of the energy determined by the distance (b) between the two nodes that represent the forward step. Obviously, the accuracy of this calculation is dependent on the size of this step. Presumably, the finite difference of the energy will approach the differential in the limit when the mesh in front of the crack becomes infinitely dense. However, the error of the energy release calculation is still not zero in that case because the energy calculations also depend on the discretization error of the overall mesh. We have not made an attempt to analyze the question of the discretization error here. It is clear, however, that the mesh distribution should be such that element size in front of the crack tip is small. At present, how small, can only be determined on the basis of experimentation and comparison with well established benchmark problems.

\section{Case Studies}

5.1 A rectangular plate with a diagonal crack

To verify the correctness of the procedure, we first tested the release rate calculation on a well known basic example that is defined in figure 5 . This is the case of a slanted crack in a plate in tension. We compared our results with analytical solutions presented in the literature in terms of the $K_{I}$ and $K_{I I}$ factors e.g., Stern, Becker, and Dunham (1976). The agreement was:

$$
\frac{\mathrm{K}_{\mathrm{I}}}{\mathrm{K}_{\mathrm{I}}^{*}}=.9877 \quad \frac{\mathrm{K}_{\mathrm{II}}}{\mathrm{K}_{\mathrm{II}}}=.9787
$$

where the asterix denotes the values obtained by the analytical method. The results were obtained for a mesh that is given in figure 6.

\subsection{A longitudinal crack in a pressurized fuselage}

A segment of a barrel fuselage section of a design that is representative of a present day airliner is pictured in figure $7^{b}$. It is a subsection of a much larger finite element model of a fuselage section that is part of an extensive damage tolerance analysis program: Starnes (1992), figure 7a. The global model is used to study the disturbance of the stress distribution in the super structure that is caused by these "large" cracks. The loading on both models is the cabin pressure during flight. 


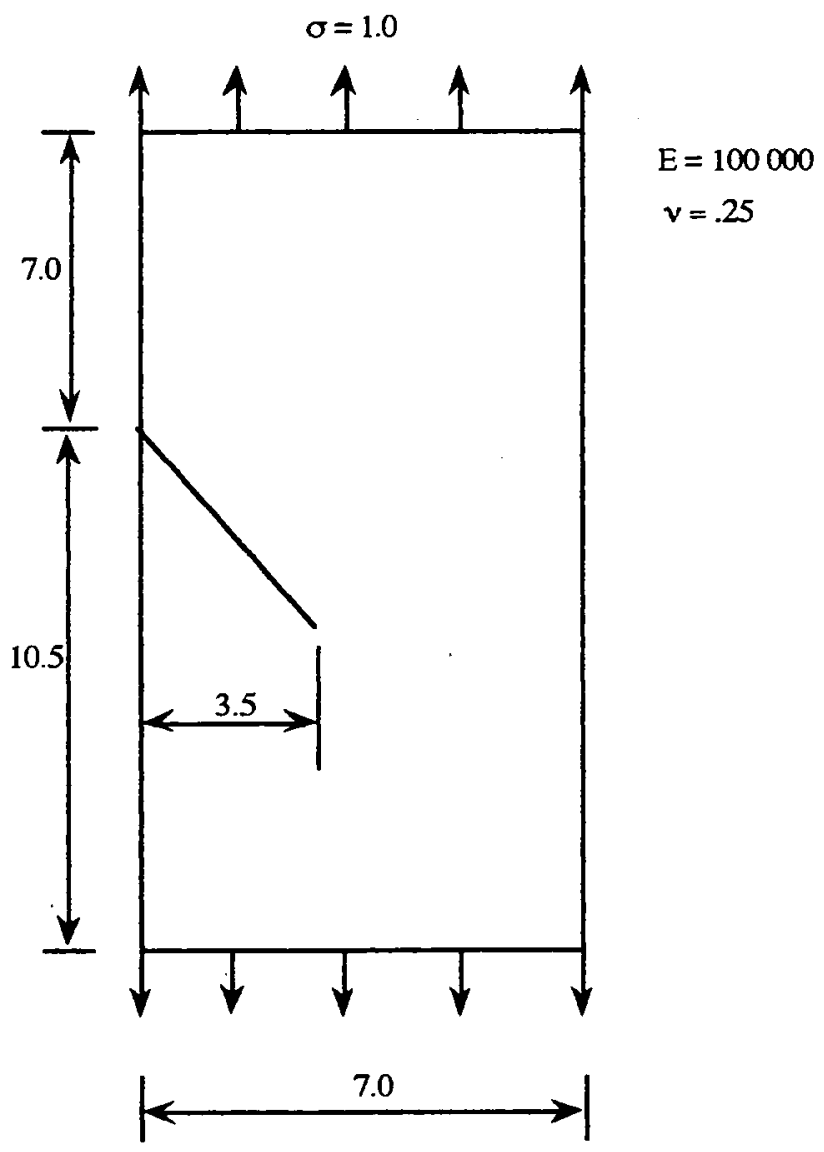

\section{Slanted Crack Panel}

Figure 5 Slanted Crack

The ring stiffeners and longerons of our panel model are modeled by shell elements. The objective of the analysis is to evaluate the behavior of a longitudinal crack that is symmetrically positioned between two bulkheads and two longerons for values of the crack length varying between 10 . to 20 . inches.

The mesh that we used to solve this problem is pictured in figure $7^{\mathrm{b}}$. It leads to a set of equations of a dimension of about 15000 degrees of freedom. We solved the problem partly on a Cray II, partly on a Titan mini super computer to shorten the turn around time. It can, however, also be carried out on any fast work station with sufficient backup memory. The problem was analyzed for a number of crack lengths $2 \mu$; i.e., $2 \mu_{1}=10.21,2 \mu_{2}=15.21 ; 2 \mu_{3}=20.21$.

Because the cracks are rather long, there is considerable interaction between the crack behavior and the response of the surrounding structure. This interaction requires a reformulating of the boundary conditions for our model for each new crack geometry. In other words, when the 
length of the crack changes, the boundary conditions also change. The changes are computed from the global model. Consequently, in this particular investigation it was necessary to recompute the loading sequence for each of the geometries of the crack that we considered in the analysis (this corresponds to the computation of curves of the type $A_{0}-A$ and $B_{0}-B$ in figure 2.).

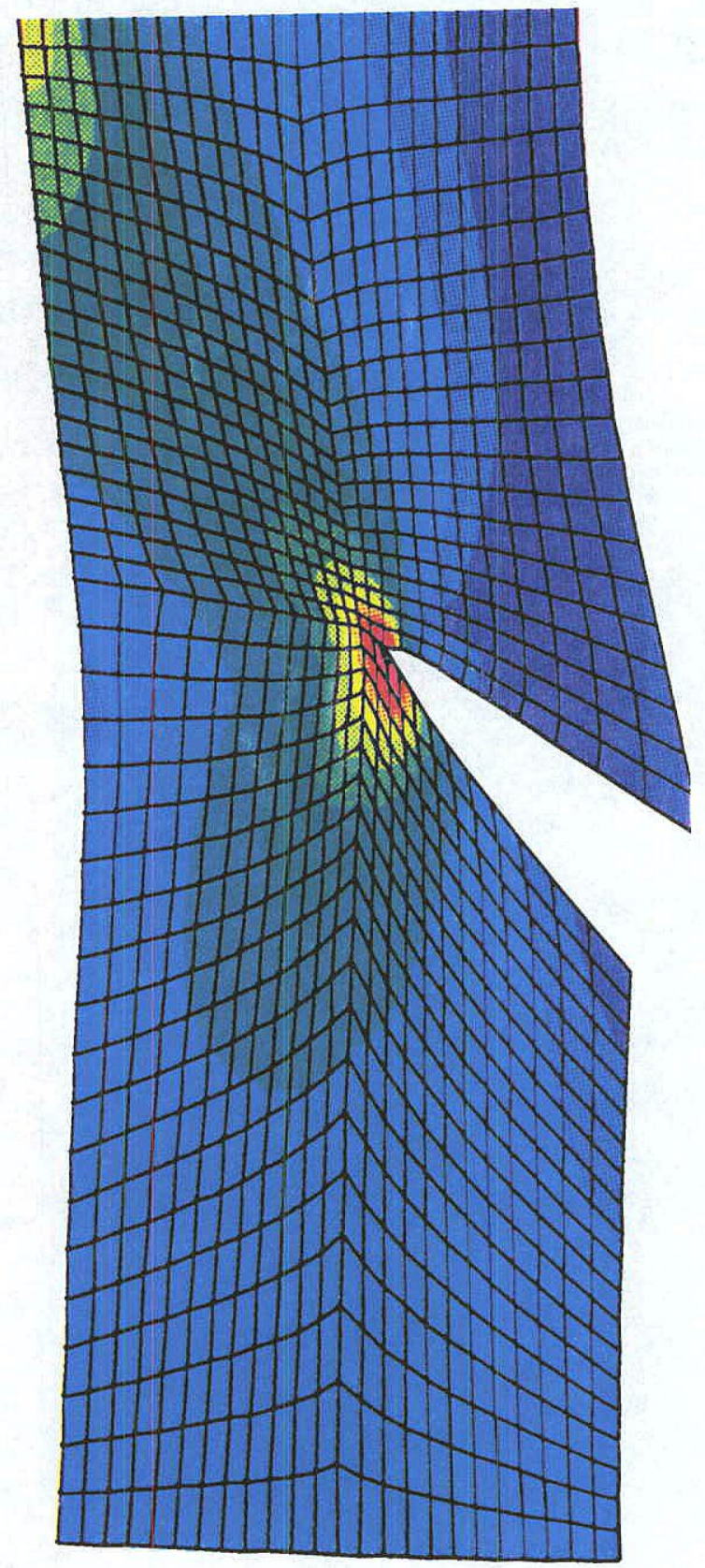

Figure 6 Diagonally - Cracked Plate

At each final deformation state for the service load $(\lambda=1)$ that were obtained, we determined the energy release rates by the method of node release. At the same time we also computed the global energy release rates by means of the discrete analog of the $\mathrm{J}$ - integral method mentioned earlier (which is also known as the virtual crack extension method, e.g. Parks (1974), Hellen (1975), deLorenzi (1987)). 


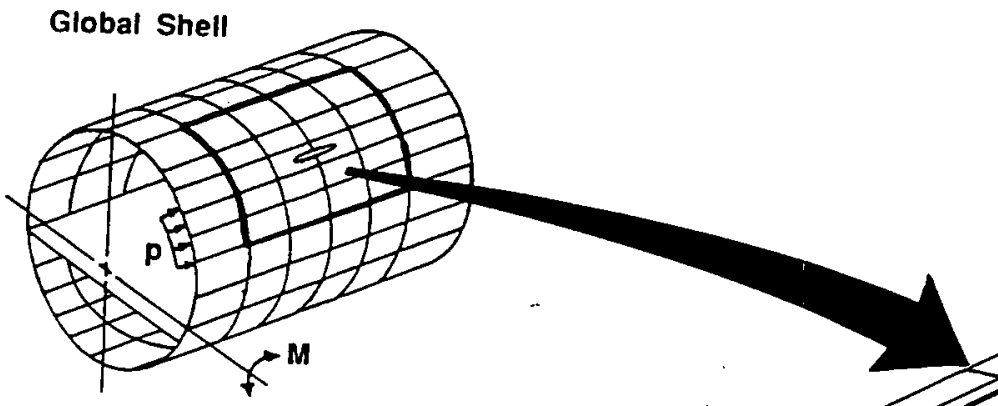

Stiffened panel

- Beams for stringers and floor beams

- Plates for frames

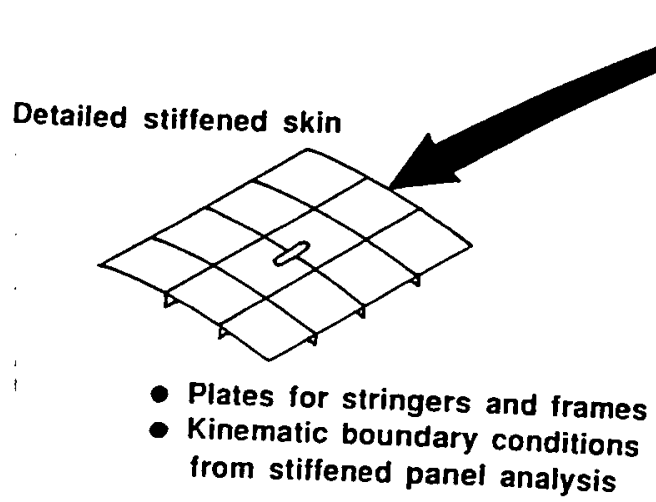

Figure $7^{\mathbf{a}}$ Hierarchical shell modeling strategy

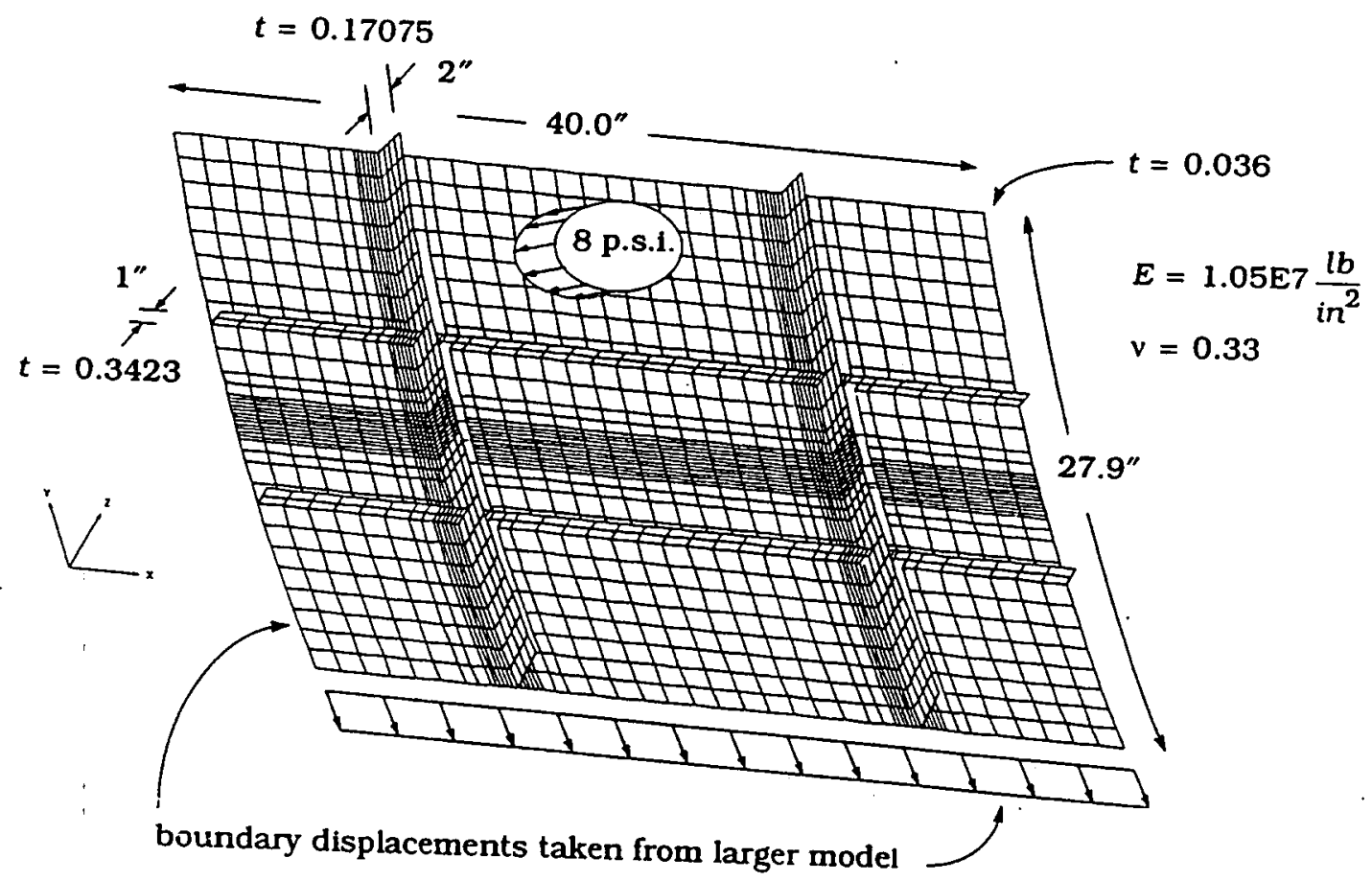

Figure $7^{\mathrm{b}}$ Model geometry for pressurized fuselage section 
HOOP STRESS FOR 15 INCH CRACK

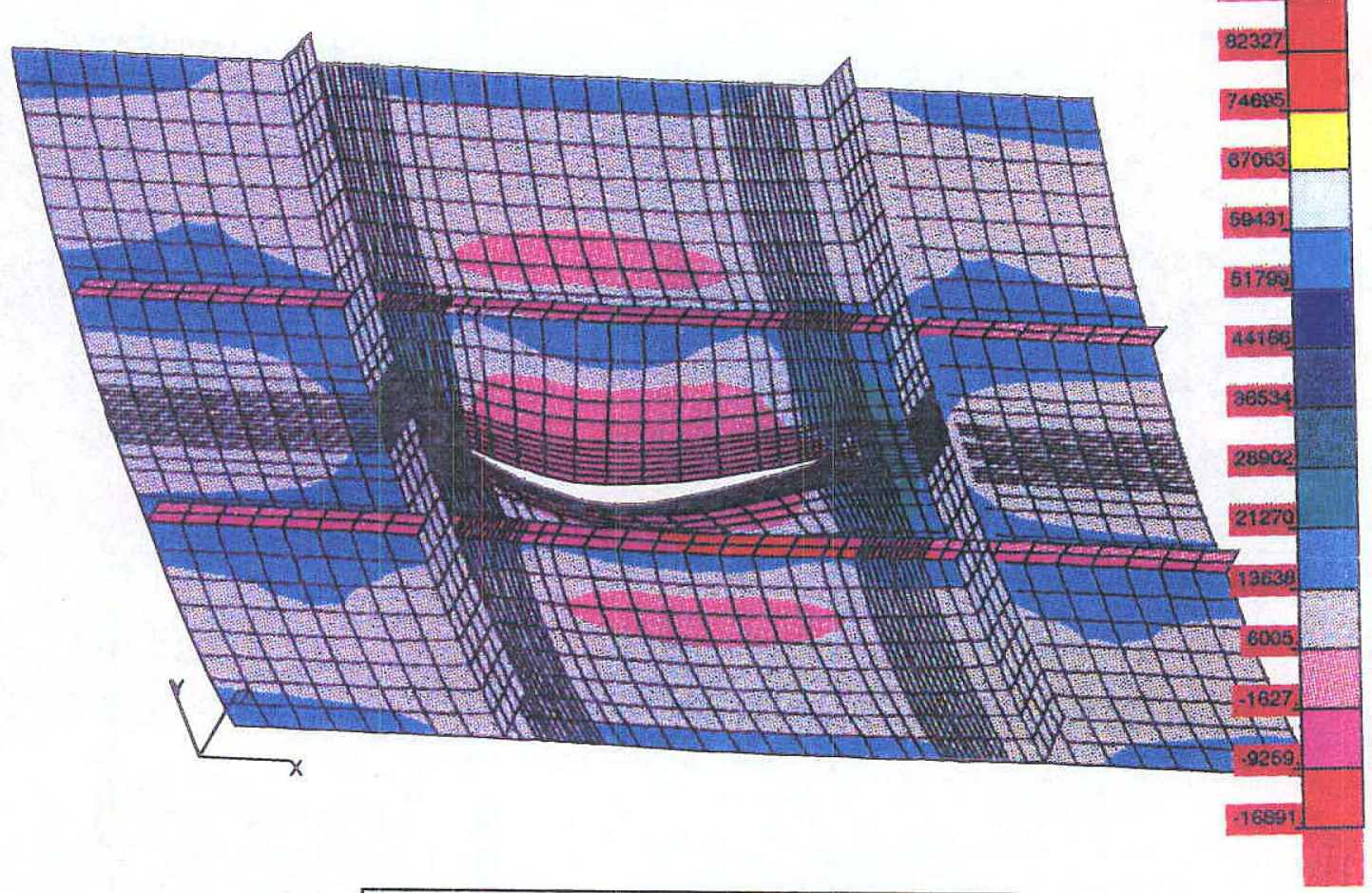

Figure 8 The fifteen inch crack model

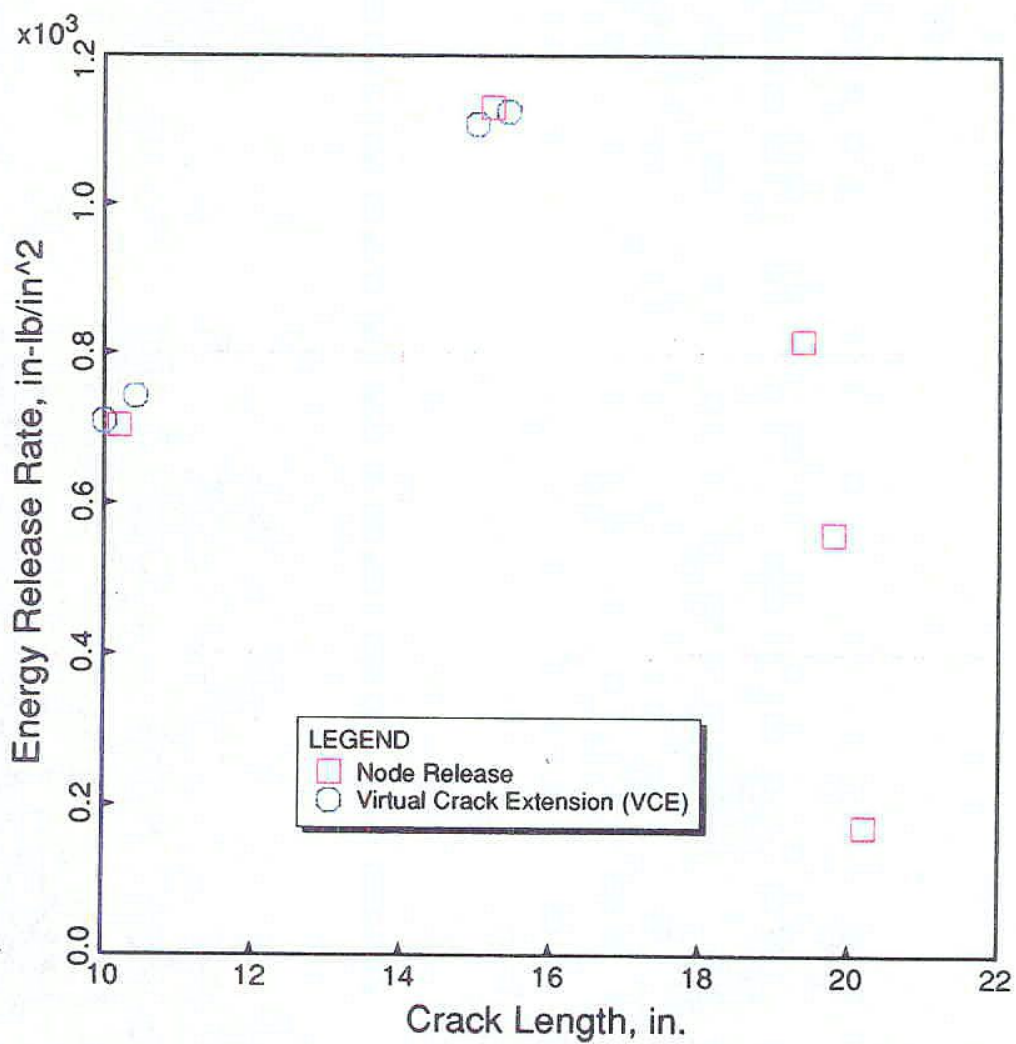

Figure 9 Midbay crack energy release rates: VCE vs. NR 


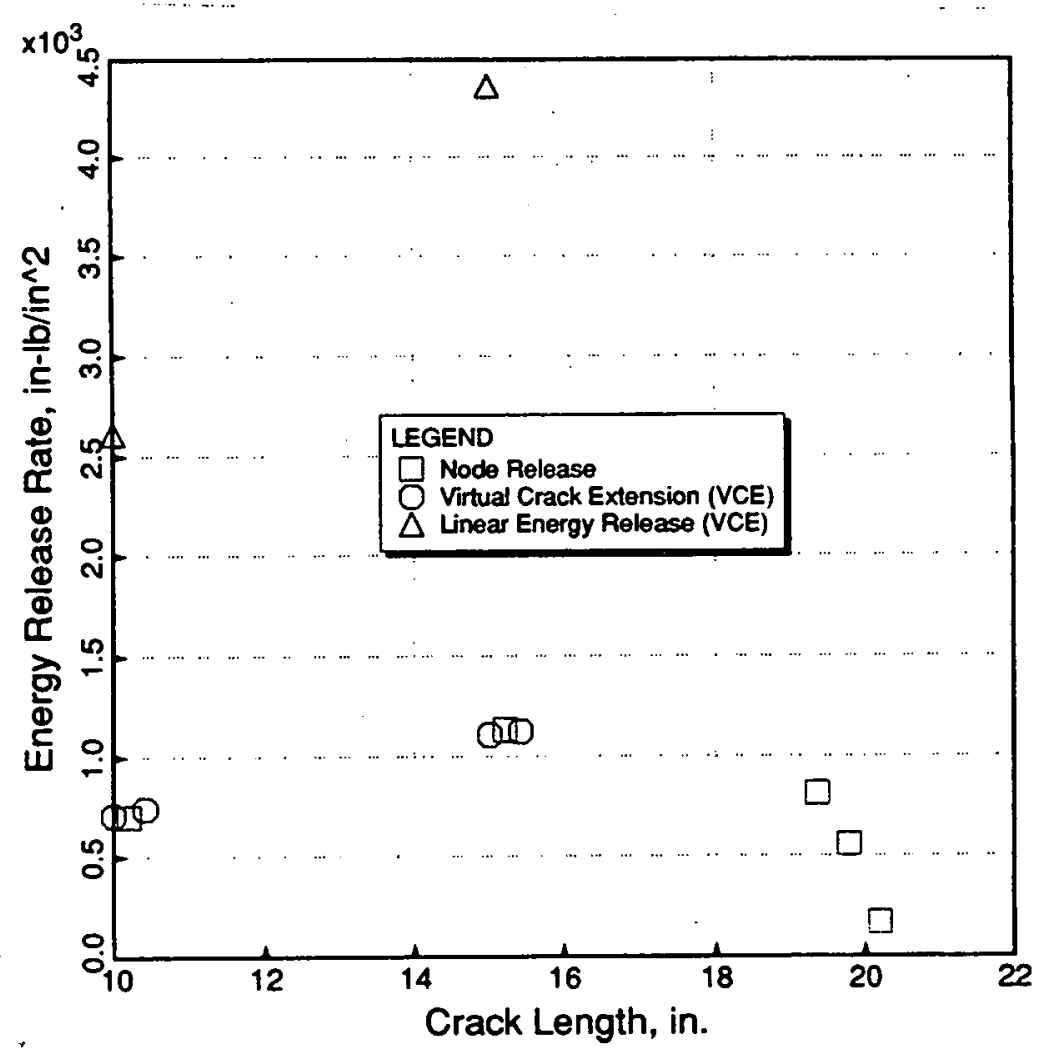

Figure 10 Midbay crack energy release rates: nonlinear vs. linear

Crack Length (in) Method Mode $\underline{G\left(i n-l b / i n^{2}\right)}$

$\begin{array}{llll}10.00 & \text { VCE } & I_{m} & 709 \\ 10.21 & \text { NR } & I_{m} & 702 \\ 10.42 & \text { VCE } & I_{m} & 742\end{array}$

15.

15.21

15.416

VCE

$I_{m} \quad 1107$

NR $\quad I_{m} \quad 1130$

VCE $\quad I_{m} \quad 1123$

19.375

NR

$I_{m}$

817

20.21

NR

$I_{m}$

126.5

20.21

NR

$I I_{m} \quad 18.0$

20.21

NR

$I I I_{m} \quad 11.8$

20.21

NR

$I I_{b} \quad 14.5$ 
The results of the calculations are tabulated in table 1. As can be seen, the results of the two methods are in close agreement. We can also see that for the largest crack analyzed, the cracking mode is no longer restricted to mode I only, and this shows the importance of influence of the ring stiffener on the loading of the crack. In figure 9 and 10 the mode I energy release rate is plotted against the crack length $2 \mu$. The first picture gives a very clear account of the alleviating effect the ring stiffener has on the loading of the crack when the crack front approaches the ring stiffener (or bulkhead). Figure 10 presents the same data that are displayed in figure 9 , but now on a smaller scale. In this graph we also included a data point that corresponds to a linearized analysis. It very clearly illustrates that linear shell theory is definitely inappropriate for the analysis of the bulging of the cracks considered here .

\section{Conclusion}

\subsection{Conclusions}

The tools of analysis we described in this paper are fairly easy to apply and their application is not hampered by the complexity of the model in which the crack(s) reside. As compared with other methods, the drawback of the node release method for the computation of the energy release rates concerns mainly the fine mesh that needs to be applied at the crack tip in order to maintain accuracy. In all other aspects, as we believe to have shown, the method is simple, robust and accurate enough for most engineering applications. What seems to us of particular importance is the possibility to apply this method of calculation to cracks in the fuselage skin that are growing towards and underneath a stiffener.

\section{References}

1. Lemaitre, J., Turbat, A. and Loubet, R. (1977): Fracture Mechanics Analysis of Pressurized Cracked Shallow Shells, Engrg. Fract. Mech., 9, 443-460.

2. Riks E., den Reijer PJ. (1987), A Finite Element Analysis of Cracks in a Thin Walled Cylinder under Internal Pressure, National Aerospace Lab. NLR, The Netherlands, NLR TR $87021 \mathrm{U}$.

3. Riks E.(1987), Bulging Cracks in Pressurized Fuselages: A Numerical Study, National Aerospace Lab. NLR, The Netherlands, NLR MP $87058 \mathrm{U}$.

4. Ansell, H. (1988), Bulging of Cracked Pressurized Aircraft Structure, Ph.D. Thesis, Linkoping Institute of Technology, Sweden.

5. Atluri S. and Pin Tong (1991), Computational Schemes for Integrety Analysis of Fuselage Panels in Aging Airplanes, Springer Verlag Berlin Heidelberg, Springer Series in Comp. Mechanics, Atluri, Sampath and Tong (eds.).

6. Chen D. (1991), Bulging Fatigue Cracks in a Pressurized Aircraft Fuselage, Delft University, Dept. of Aeronautics \& Astronautics, Delft, The Netherlands, PhD.Thesis. 
7. Chen, D. and Schijve J.(1991), Bulging of Fatigue Cracks in a Pressurized Fuselage,

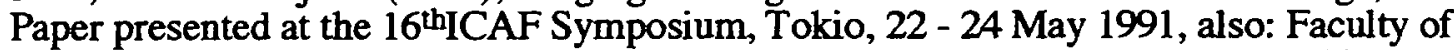
Aerospace Engineering, Delft University, Delft, The Netherlands, Report LR - 655.

8. Riks E., Brogan F.A., \& Rankin C.C. (1989), Bulging Cracks in Pressurized Fuselages: A Procedure for Computation, December Meeting ASME, San Francisco. In: Analytical and Computational Models of Shells, Proceedings Winter Annual Meeting ASME (A.K. Noor, T. Belytschko, J.C. Simo eds.), C.E.D. Volume 3, The American Society of Mechanical Engineers.

9. Riks E., Rankin C.C.\& Brogan F.A.(1991), The Buckling Behavior of a Central Crack in a Plate under Tension, Faculty of Aerospace Engineering, Delft University, Delft, The Netherlands, Report LR - 667, To appear in Enrgn. Fract. Mech..

10. Almroth B.O., Brogan F.A. \& Stanley G.M.(1983), Structural Analysis General Shells, Lockheed Missiles and space Co., Palo alto, California, Vol.2, Users Instructions for STAGSC-1, Report LMSC D633873.

11. Finnie I. and Saith A. (1973), A Note on the Angled Crack Problem and the Directional Stability of Cracks, Int. J. of Fract., 9, 484-486.

12. Leevers P.S., Radon J. C. and Culver L. E. (1977), Fracture Trajectories in Biaxially Stressed Plate, J. Mech. Phys. Solids, 24, 381-395.

13. Cotterell B. and Rice J. R.(1980), Slightly Curved or Kinked Cracks, Int. J. of Fract., 16, No. 2.

14. Sumi Y., Nemat- Nasser S. and Keer L. M. (1985), On Crack Path Stability in a Finite Body, Engrg. Fract. Mech., 22, No. 5, $759-771$.

15.Rubinstein A. A. (1991), Mechanics of the Crack Path Formation, Int. J. Fract., 47, 291305.

16.Ramulu M., Kobayashi A. S., Kang B. S. (1982), Dynamic Crack Curving and Branching in Line- Pipe, Journal of Pressure Vessel Technology, 104/317

17.Ramulu M., Kobayashi A. S. (1983), Dynamic Crack Curving - A Photoelastic Evaluation, Experimental Mechanics, 23, 1 - 9.

18.Zaal K. J. J. M, (1992), A Survey of Crack Path Stability Theories with an Eye to an Application to Crack Flapping Phenomena in Pressurized Fuselages, Faculty of Aerospace Engineering, Delft University, Delft, The Netherlands, Report LR - 681.

19. Koiter W. T. (1966), On The Nonlinear Theory of Thin Elastic Shells, Proc.Kon. Ned. Ak. v. Wetenschappen, ( Proc. Royal Dutch Society of Science), 1- 54.

20. Riks E. (1984), "Some Computational Aspects of The Stability Analysis of Nonlinear Structures", Comp. Meth. in Appl. Mech. and Engrg., 47, 219-259.

21. Riks E. (1987), Progress in Collapse Analysis, Journal of Pressure Vessel Technology, $109,27-41$.

22. Rankin C. C. and Brogan F. A. (1991), The Computational Structural Mechanics Testbed Structural Element Processor ES5: STAGS Shell Element,Lockheed missiles \& Space CO., Palo Alto, California, NASA Contractor Report 4358.

23. Niordson F. I. (1985), Shell Theory, Amsterdam: North - Holland. 
24. Parks D.M. (1974), A Stiffness Derivative Finite Element Technique for Determination of Elastic Crack Tip Stress Intensity Factors, Int. J. Fract., 10, No. 4, 487-502.

25.Hellen T.K.(1975), On the Method of Virtual Crack Extensions, Int. J. Num. Meth. Engrg., 9, No.1, 187-207.

26.deLorenzi H.G. (1985), Energy Release Rate Calculations by the Finite Element Method, Engrg Fract. Mech., 21, No. 1, 129.

27.Hellen T. K., Blackburn W. S. (1987), Nonlinear Fracture Mechanics and Finite Elements, Eng. Comp., 4, 2 - 14.

28.Stern M., Becker E. B., and Dunham R. S. (1976), A Contour Computation of Mixed Mode Stress Intensity Factors, Int. J. Fracture, 12, No 3.

29.Starnes J. and Britt V. (1992), Nonlinear Analysis of Stiffened Fuselage Shells with Local Damage, Atlanta, GA, USA, paper presented at: The International Workshop on Structural Integrity of Aging Airplanes. 


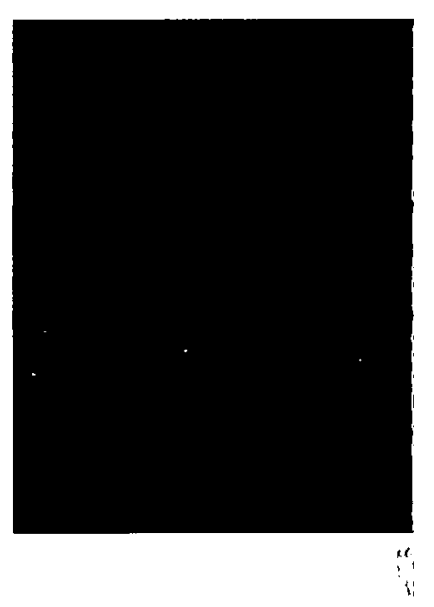

927195 